Rubus foliolosus Hal. a. a. O. S. 265 in $\boldsymbol{R}$. foliolatus, da bereits ein $\boldsymbol{R}$. pauciftorus Wall. und $\boldsymbol{R}$. foliolosus Don existirt.

Wi $ө$, im Mai 1891.

$$
\text { Dr. F. v. Halácsy. }
$$

\title{
Rathschläge für Phytographen, insbesondere für die auf dem Gebiete der Kryptogamenkunde,
}

\section{Von P. A. Saccardo.}

Die ausgedehnten Erfahrungen, welche ich bei der Ausarbeitung meiner "Sylloge fungorum omnium" gewonnen habe, brachten mir die Ueberzengung von der Nützlichkeit, ja ich kann sagen, der Nothwendigkeit, bei der Beschreibung der Pflanzen gewisse nur zu oft vernachlässigte Regeln zu befolgen. Folgende seien hiermit empfohlen:

1. Es ist nothwendig, dass die Botaniker. welche nene Species vom morphologischen und biologischen Standpunkte aus mit minutiösen und sehr verwickelten Einzelheiten beschreiben, damit knappe und vergleichbare Diagnosen der Art oder Gattung (am besten lateinisch) nach den phytographischen Regeln verbinden. In der That ist es sehr schwierig und oft sehr unsicher, in der Menge von Einzelheiten die wesentlichen und unterseheidenden Merkmale herauszufinden.

2. Die Diagnose ist bei gewissen Autoren (insbesondere anf dem Gebiete der Kryptogamen) ausserordentlich detaillirt und weitschweifig, bei anderen zu lakonisch. Eine gute Diagnose soll in knapper und klarer Form nur die wesentlichen und unterscheidenden Merkmale angeben; jede Bemerkung über Einzelheiten soll orst nach der Diagnose gegeben werden; für die veuen Arten ist es auch nothwendig, ihre Verwandtschaft mit den bekannten nächstverwandten anzugeben. Wer neue Arten bestimmt, weiss, wie viel Zeit dies kostet, wenn er es mit sehr weitschweifigen Diagnosen obne Angabe der Verwandtschaft zu thun hat.

3. Die Erfahrung hat bereits gezeigt, wenigstens für die Kryptogamen, dass es hinsichtlich der Autorenbezeichnung sehr nützlich ist, in Klanmern den Autor anzugeben, welcher zuerst die betreffende Art unter anderen Gattungen beschrieben hat. Es ist immer nothwendig, den Namen des Autors beizufügen, welcher die Art aus der ursprünglichen Gattung in eine andere versetzt hat; denn andernfalls müsste man annehmen, dass der Verfasser der Abhandlung, in welcher die Combination der Namen angeführt wird, auch der Antor dieser Combination sei. Wir finden z. B. in den Schriften Winter's Namen wie folgende: „Sphaerella convexula (Schwein.) Syn. Sphaeria convexula Schwein." Wenn wir nicht den Namen Thümen nach der Klammer beifügen, müssten wir glauben, dass Winter der Autor 
der Combination sei, und dann hätten wir nach den Regeln der übrigen Botaniker die beiden Ausdrücke: Sphaerella convexula (Schwein.) Wint. oder Sphaerella convexula Wint., welche beide falsch sind. Wenn wir aber sagen Sphaerella convexula (Schwein.) Thüm., so haben wir die ganz genaue Angabe, dass Schweinitz die Art aufgestellt und Thümen sie in die richtige Gattung versetzt hat.

4. Bei der Beschreibung parasitischer Kryptogamen sind die Nährpflanzen (oder -Thiere) mit deren technischer lateinischer Nomenclatur anzuführen. Die Vulgärnamen (englisch, italienisch, deutsch ete.) sind oft schwierig zu identificiren.

5. Für die Maasse der Organe, sowohl mikroskopische als makroskopische, ist ein einheitliches Maass, nnd zwar das metrische anzuwenden; für die mikroskopischen Maassangaben seien die Mikromillimeter oder micra $(\mu)$ an Stelle der Brüche empfohlen; die verschiedenen Masse und die Brüche sind sehr oft die Quelle von Irrthümern oder Zweifeln.

6. Zur kurzen Angabe der Dimensionen mikroskopischer Organe empfiehlt es sich (wie es übrigens schon vielfach geschieht), zuerst die Ziffer für die Länge zu setzen, hieranf jene für die grösste Breite, beide verbunden durch das Zeichen $=$, und das Zeichen $\mu$ wegzulassen; für flachgedrückte Organe kann man noch eine dritte Ziffer für die Dicke beifügen; z. B. Spore $15=4$ bedeutet: Spore $15 \mu$ lang, $4 \mu$ breit und dick; Spore $15 \smile 4 \smile 2$ bedeutet: Spore $15 \mu$ lang, $4 \mu$ breit und $2 \mu$ dick. Mehrere Autoren wenden statt des Zeichens $=$ (welches ich seit 1872 vorgeschlagen und angewendet babe) die Zoichen $=,:, \times$ an, welche in der Mathematik einen anderen und bestimmten Sinn haben. Für die makroskopischen Organe wird man die Maasseinheit, z. B. $\mathrm{m}, \mathrm{cm}, \mathrm{mm}$ und die gemessene Partie anzugeben haben.

7. Bei der Bezeichnung aller Pfanzengruppen wendet man Fominina an (Dicotyledones, Ranunculaceae, Anemoneae, etc.), man sollte das Gleiche für die Kryptogamen thun; wenn wị also sagen Sphaeriaceae, Mucedineae, Hydneae, sollten wir nothwendigerweise auch sagen Pyrenomyceteae, Hyphomyceteae, Hymenonyceteae und nicht Pyrenomycetes, Hyphomycetes, Hymenomycetes, wie Viele thun.

8. Die Farben der Pflanzen, besonders jene der Blumenkronen, der Pilze, der Sporen, werden oft mit unbestimmten Bezeichnungen beschrieben. Es wäre gut, eine bestimmte Nomenclatur anzuwenden, welche sich auf Normal-Exemplare stützt. Ich werde zu diesem Zwecke eine Chromotaxia ) publiciren, welche, wie ich hoffe, von grossem Nutzen sein wird.

9. Was die Nomenclatur der Früchte und Sporen der Pilze betrifit, so wäre es nützlich, nur die folgende anzuwenden, welche übrigens von der Mehrzahl der Mykologen angenommen ist. Hymenomyceteae: Pileus (welche Form er auch besitze); badisia; sterigmata; sporae; cystidia.

y) Vergl. S. 178. 
Gasteromyceteae et Myxomycetea 0 : Peridium; gleba; capillitium; floci; sporae.

Uredineae: Sorus; uredosporae; teleutosporae; mesosporae; pseudoperidium; aecidiosporae; paraphyses.

Ustilaginoae: Sorus; sporae.

Phycomyceteae: Oogonia; oosporae; antheridia ; spermatia; zygosporae; azygosporae; zoosporangia; zoosporae.

Pyrenomyceteae et Phymatosphaeriaceae: Stroma; perithecium; loculus; ascus; sporidia; paraphyses.

Discomycetea et Tuberoideae: Ascoma; gleba; ascus; sporidia; paraphyses.

Schizomyceteae: Filamenta; baculi; cocci; endosporae; arthrosporae.

Sphaeropsidea: Perithecium; basidia; sporulae.

Melanconieae: Acervulus; basidia; conidia (aber nicht gonidia, ein Name, der für die Flechten reservirt bleiben muss).

Hyphom yceteae: Caespitulus; sporodochium; hyphae; sporae.

An merkung: Aus der keimenden Spore entsteht das promylecium, welches im Allgemeinen die sporidiola producirt.

\section{Litteratur-Uebersicht.')}

April 1891.

Beck G. v. Versuch einer neuen Classification der Früchte. (Abh. d. zool.-bot. Ges. XLI. S. 307.) 80. 6 S.

Dominicus M. Einige Pflanzenstandorte in der Umgebung Voitsbergs. (Mitth. d. naturw. Ver. f. Steiermark 27.) $8^{\circ} .18$ S.

Entleutner A. Die immergrünen Ziergehölze von Südtirol. München (M. Huttler). 80. 173 S., 114 Abb. auf 73 Taf. nach Foderskizzen des Verf, 8 Lichtdruckbilder nach Photographien.

Filarszky N. A kikeleti horivág nehány virágalakjáról, Galanthus nivalis. (Termeszet. füz. XIIL. P. IV. p. 110-113.) 8".

Deutsche Uebersetzung auf S. 173-176: Ueber Blüthenformen bei dem Schneeglörkchen.

Halácsy F. v. Oesterreichische Brombeeren. (Abh. d. zool.-bot. Ges. XLI. S. 197.) $8^{\circ} .98 \mathrm{~s}$.

1) Die „Litteratur-Uebersicht* strebt Vollständigkeit nur mit Rücksicht auf jene Abhandlungen an, die entweder in Oesterreich-Ungarn erscheinen oder sich auf die Flora dieses Gebietes direct oder indirect beziehen, ferner auf selbstständige Werke des Auslandes. Zur Erzielung thunlichster Vollständigkeit werden die Herren Autoren und Verleger um Einsendung von neu erschienenen Arbeiten oder wenigstens um eine Auzeige über solche höflichst ersuclit.

Die Red. 